\title{
Excel Files for Teaching About Wave Fronts and Rays for a Wave Moving in Media with Changing Index of Refraction
}

\author{
Pavlos Mihas \\ The Department of Primary Education, School of Education, Democritus University of Thrace, Alexandroupolis, Greece \\ Email address: \\ pmichas@eled.duth.gr \\ To cite this article: \\ Pavlos Mihas. Excel Files for Teaching About Wave Fronts and Rays for a Wave Moving in Media with Changing Index of Refraction. \\ American Journal of Physics and Applications. Vol. 9, No. 4, 2021, pp. 94-101. doi: 10.11648/j.ajpa.20210904.14
}

Received: August 10, 2021; Accepted: August 20, 2021; Published: August 27, 2021

\begin{abstract}
In teaching about waves the students are learning how to find the refracted rays by using wave fronts or finding the wave fronts by using the rays. The usual teaching is based on models where the speed of the wave is constant in one medium and changes abruptly as the wave passes from one medium to another. This paper deals with ways of calculation the wave fronts and the rays for the case of a continuous changing of the index of refraction. For this purpose, Fermat's principle is applied for multiple layers of very small thickness. Two models are presented for the speed of the waves: A model on which the wave speed depends on the square root of the depth of penetration of the wave and the other model, where the speed depends on a linear dependence. In both cases it is found that as the wave progresses it is "totally reflected". In the case of the "square root dependence" the solution is a kind of cycloid which shows this behavior. In the linear case it is found that there is a moment where the wave is reflected, which is found by the maximum of a quantity " $Z$ ". By using this quantity, the coordinates $\mathrm{x}$ and $\mathrm{y}$ can be calculated. As an application the refraction of the light in the atmosphere is calculated, where the dependence of the distance from the center of the earth is calculated and again the 2 models are applied. In this case the "square root" model gives a stronger deviation from the linear model. This helps the student to understand the change on the perceived position of the celestial bodies.
\end{abstract}

Keywords: Rays, Wave Front, Snell' Law, Total Reflection

\section{Introduction}

Science is primarily concerned with the description and explanation of physical patterns in the universe, from the astronomical scale to the subatomic scale. Scientists carry out their tasks through modeling inquiry. They construct, corroborate, and deploy conceptual models each of which represents a particular pattern in certain respects and to a certain extent, and serves a particular descriptive and/or explanatory function regarding the pattern in question. Scientific models and modeling can be transposed into pedagogical tools and processes which students of all levels can efficiently take advantage of for meaningful and sustainable learning of scientific theory.

The merits of models and modeling processes are being increasingly recognized not only in scientists' work, but, most importantly, in human cognition in general and in science education in particular. [1]

For physics education simulations are of great importance.
Spreadsheets give the opportunity for integrating computational physics techniques with simulation and traditional teaching learning methods of Physics, a major improvement in the conceptual understanding of students is expected [2]. There are examples of using Excel simulations in Kinematics [3, 4] Optics is very well suited for using spreadsheets [5-7].

Teaching about wave fronts in introductory courses is usually restricted to the teaching of the refraction of periodic waves in media that have a constant speed as for example [8]. This paper was based in the ideas expressed by [9] in the tutorials of introductory physics. In Pavlos Mihas and Triantafyllos Gemousakakis's study, these ideas were expanded for teaching introductory courses by using rays and wave fronts in a medium in which the index of refraction changes [10]. The rays and wave fronts in a medium with an index of refraction that changes can help the students to understand these notions. In this paper are presented ways to use Excel files without macros to teach about waves and rays 
with a changing speed.

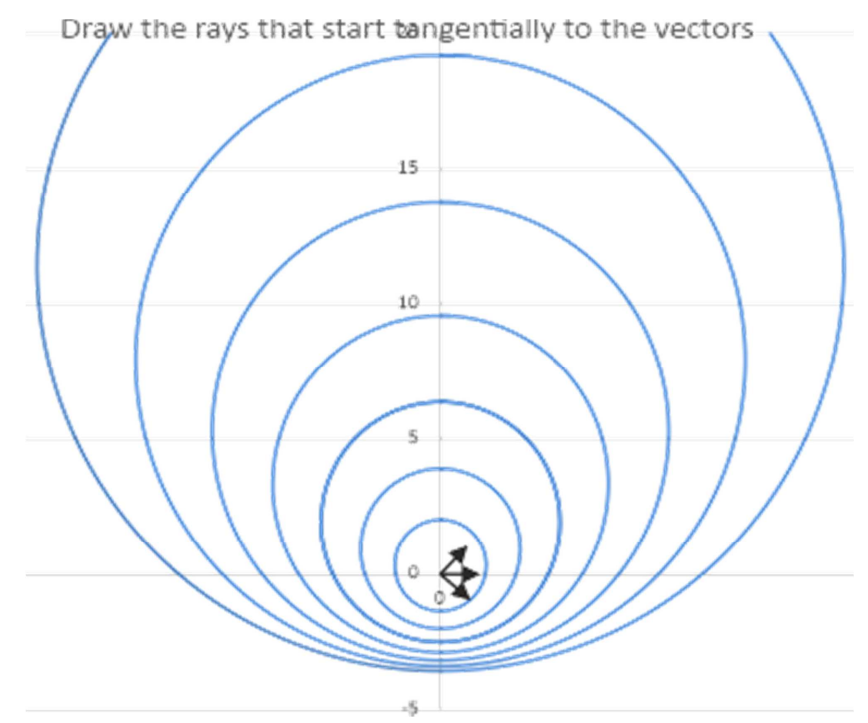

Figure 1. A question for exams: draw the rays that start along the arrows.

One of the first solutions of this problem it was done by Jean Bernoulli $[11,12]$. He was interested in solving the problem of "brachistochrone." If we imagine a curve on a coordinate system with the x-axis horizontal, and the y-axis vertically downward. When the material point sliding down the curve passes a certain point $(\mathrm{x}, \mathrm{y})$ with a certain velocity v. We have the relation: $\frac{v^{2}}{2}=g y$ which was familiar to Bernoulli. So, $\mathrm{v}=(2 g y)^{\frac{1}{2}}$. Bernoulli solved the problem by using Fermat's principle. He proposed that the speed of the wave changes with square root of $\mathrm{y}$. and then finds the curve from A to B as a cycloid. This was a solution of the problem of the brachistochrone. As Polya points out the mechanical problem admits an optical reinterpretation. The medium in which the light is transmitted is an optically inhomogeneous medium. This medium is stratified, has strata of different index of refraction; the velocity of light in the horizontal layer at the depth y is $(2 g y)^{\frac{1}{2}}$.

In this paper we compare models of propagation of waves. Model comparison is at the heart of all scientific methodologies [13]. Progress is made in science by constructing many models (possibly of different complexities), testing them against measurements, and determining which of them explain the data the best.

\section{Models of Refraction}

Two models are presented for the speed of the wave:

A) A model with the velocity being equal to $\sqrt{A+\beta y}$ where $A$ and $\beta$ constants, and b) A model with the velocity being equal to $A+B y$

We then apply the method to a ray coming from the moon. The movement of light in the atmosphere was dealt first time by Newton (Nauenberg 2017) who dealt on the movement of the particles of light. We apply methods similar to the ones who are used in the movement of light, but we have to solve for the $(r, \theta)$ coordinates. The speed is given by a square root.

If we consider a medium in which the speed of light (or more generally of a wave) depends on the y-coordinate as $v=\sqrt{A+\beta y}$ then Snell's law is given by the constancy of $\frac{\sin \alpha}{v}=\Gamma$ where $\Gamma=\frac{\sin \alpha_{0}}{v_{0}}$ where $\alpha_{0}$ is the initial angle of incidence. The angle $\alpha$ is related to the angle of inclination $\theta$ by $\alpha=\pi / 2-\theta$ or $\sin \alpha=\cos \theta$ since $\tan \theta=\frac{d y}{d x} \sin \alpha=$

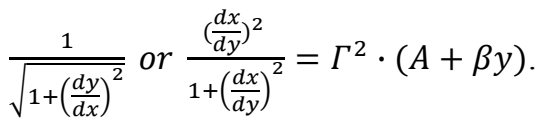

$$
\begin{gathered}
x=\int_{0}^{x} d y \sqrt{\frac{\Gamma^{2}(A+\beta y P}{E-\beta \Gamma^{2} y}} E=1-\beta \Gamma^{2} A(1) x=\frac{1}{\beta \Gamma^{2}}\left[\varepsilon-\frac{\sin 2 \varepsilon}{2}\right]_{\varepsilon_{0}}^{\varepsilon} \\
\text { where } \varepsilon=\operatorname{asin}\left(\Gamma(A+B y)^{\frac{1}{2}}\right)
\end{gathered}
$$

For the time $\mathrm{t}$ we get: For the time we ge

$$
\mathrm{t} t=\int \frac{d s}{v}=\int d y \frac{\sqrt{1+\left(\frac{d x}{d y}\right)^{2}}}{\sqrt{A+B y}}=\int d y \sqrt{\frac{1}{\left(E-\Gamma^{2} B y\right)(A+B y)}}=\frac{2}{\sqrt{\Gamma^{2} B^{2}}} \arctan \left(\sqrt{\left.\frac{B \Gamma^{2}(B y+A)}{B\left(-B \Gamma^{2} y+E\right)}\right)}\right.
$$

So solving for $y$ we get an expression of $y$ as a function of $t=\frac{2}{B \Gamma} \arctan \sqrt{\frac{\Gamma^{2}(B y+A)}{-B \Gamma^{2} y+E}}-Q$ where $Q=\frac{2}{B \Gamma} \arctan \sqrt{\frac{\Gamma^{2} A}{E}}=\frac{2 a_{0}}{|B \Gamma|}$ so $\tan \left(\frac{(t+Q) B \Gamma}{2}\right)^{12}=M=\frac{\Gamma^{2}(B y+A)}{-B \Gamma^{2} y+E}$

so $y=\frac{\varepsilon \sin ^{2} \varepsilon-\Gamma^{2} A \cos ^{2} \varepsilon}{B \Gamma^{2}}$ where $\varepsilon=\frac{(t+Q)|B \Gamma|}{2}=\frac{\left(t|B \Gamma|+2 * a_{0}\right)}{2}$

$$
\begin{gathered}
y=\frac{\sin ^{2} \varepsilon-\Gamma^{2} A}{B \Gamma^{2}}=\frac{\sin ^{2} \varepsilon-\sin ^{2} a_{0}}{B \Gamma^{2}} \\
\mathrm{x}=\frac{1}{B \Gamma^{2}}\left(\sin (2 \varepsilon)-\varepsilon-\sin \left(2 a_{0}\right)+a_{0}\right)
\end{gathered}
$$


for $\sin \varepsilon=1 y_{\max }=\frac{1-\Gamma^{2} A}{B \Gamma^{2}}=A^{2} \frac{\cos ^{2} a_{0}}{B \sin ^{2} a_{0}}=\frac{A^{2}}{B} \cot ^{2} a_{0}$ so $\varepsilon=\frac{\pi}{2}=\frac{(t+Q)|B \Gamma|}{2}$ so

$$
\begin{gathered}
t\left(y_{\max }\right)=\frac{\pi}{|B \Gamma|}-Q=\frac{\pi}{|B \Gamma|}-\frac{a_{0}}{|B \Gamma|}=2 \frac{\frac{\pi}{2}-a_{0}}{B \Gamma} \text { for } t=2 t\left(y_{\max }\right)=\frac{1}{|B \Gamma|}\left(2 \pi-2 a_{0}\right) \varepsilon=\pi-\alpha_{0} \text { so } y=0 \\
\text { for } \mathrm{t}=0 x-x_{0}=\frac{2}{B \Gamma^{2}}\left(\Gamma \sqrt{A E}-\frac{q}{1+q^{2}}\right)=\frac{2}{B \Gamma^{2}}(\Gamma \sqrt{A E}-\Gamma \sqrt{A E})=0
\end{gathered}
$$

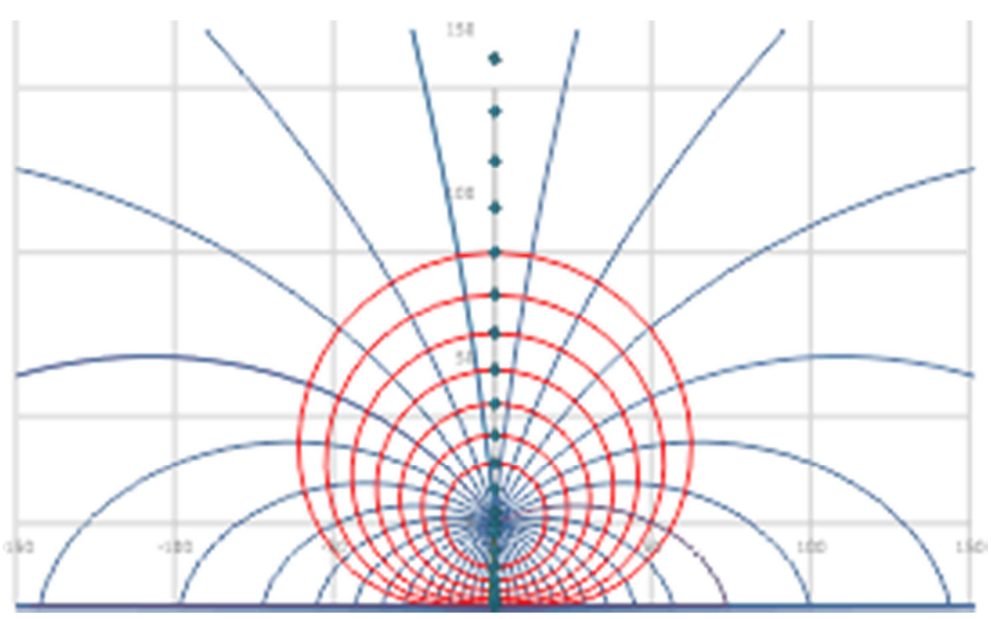

Figure 2. Case when the speed of the wave is proportional to $(A+b r)^{\wedge}(1 / 2)$.

For $a_{0}=0$ then the movement is perpendicular to the $x$-axis and then $x=0$ and we find

$$
t=\int \frac{d s}{v}=\int \frac{d y}{\sqrt{A+B y}}=\frac{2}{B}(\sqrt{A+B y}-\sqrt{A}) \frac{(B t+\sqrt{A})^{2}}{4}=A+B y \text { or } y=\frac{B t^{2}}{4}+\sqrt{A} t \frac{d y}{d t}=\frac{B t}{2}+\sqrt{A}
$$

B) If the speed is given by a linear case $v=A+B y$

Then the integration is proceeding in some respects easier:

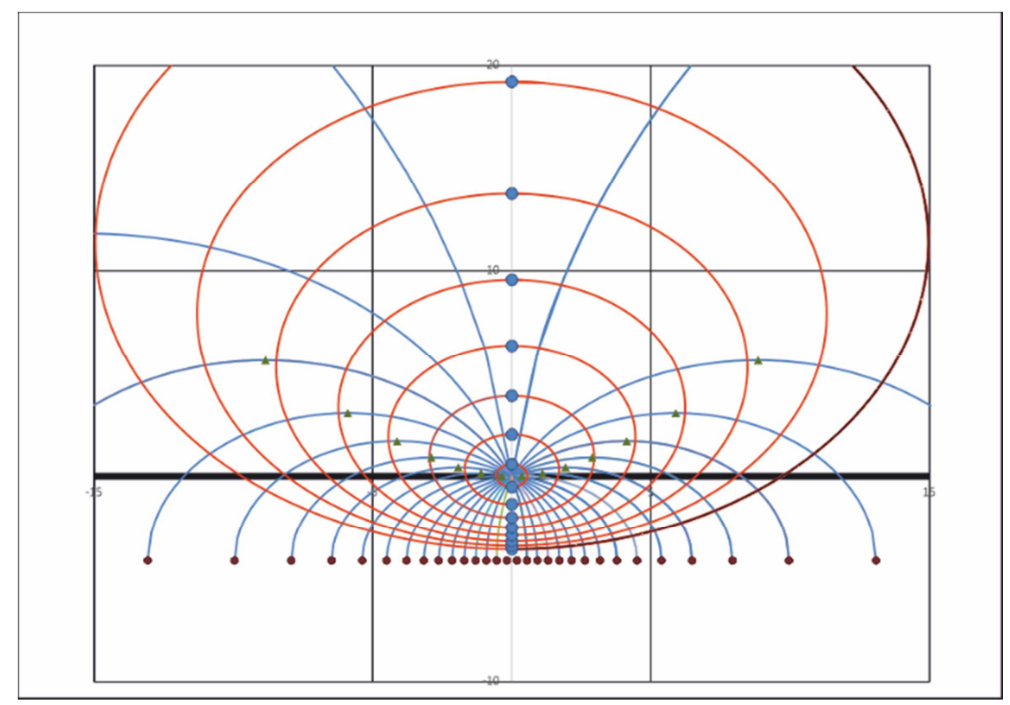

Figure 3. Rays and wave fronts if the speed is given by a linear case.

From Snell's law and $\Gamma=\frac{\sin \alpha_{0}}{v_{0}}$ we get $\frac{1}{v^{2}\left(1+\left(\frac{d y}{d x}\right)^{2}\right)}=\Gamma^{2}$ or $\frac{1}{(A+B \cdot y)^{2}}=\Gamma^{2}\left(1+\left(\frac{d y}{d x}\right)^{2}\right)\left(\frac{d y}{d x}\right)^{2}=\frac{1}{\Gamma^{2}(A+B y)^{2}}-1=$ $\frac{1-\Gamma^{2}(A+B y)^{2}}{\Gamma^{2}(A+B y)^{2}}$

$$
\left(\frac{d x}{d y}\right)^{2}+1=\frac{1}{1-\Gamma^{2}(A+B y)^{2}}
$$


By putting $\Gamma(\mathrm{A}+\mathrm{By})=\mathrm{z}$ we get:

$$
\begin{gathered}
x-x_{0}=\int_{y_{0}}^{y} d y \frac{\Gamma(A+B y)}{\sqrt{1-\Gamma^{2}(A+B y)^{2}}}=\frac{1}{2 \Gamma B} \int_{z_{0}}^{z} \frac{d z^{2}}{\sqrt{1-z^{2}}}=-\frac{1}{B \Gamma}\left(\sqrt{1-z^{2}}-\sqrt{1-z_{0}^{2}}\right) \\
\sqrt{1-z^{2}}=-\left(B \Gamma x-\cos a_{0}\right) \text { or } \\
1-\Gamma^{2}(A+B y)^{2}=\left(B \Gamma x-\cos a_{0}\right)^{2} \Gamma(A+B y)=\sqrt{1-\left(B \Gamma x-\cos a_{0}\right)^{2}} \text { so } \\
y=\frac{\sqrt{1-\left(B \Gamma x-\cos a_{0}\right)^{2}}}{\left|\sin a_{0}\right| \Gamma B}-\frac{A}{B} \text { for } x=\frac{A \cdot \cot a_{0}}{B} \frac{d y}{d x}=0 y_{m}=\frac{A}{B} \frac{1}{\left|\sin a_{0}\right|}-\frac{A}{B}
\end{gathered}
$$

For $a_{0}<\pi / 2$ or $a_{0}>3 \pi / 2$ that is the rays are moving downwards $(\mathrm{y}<0)$ the lowest point is reflection $x=-1 /(\beta \Gamma)(1-$ $\left.\left.\cos a_{0}\right)\right)=-A / B \cot \left(a_{0} / 2\right)$ and $y=-\frac{A}{B}$

The time $\mathrm{t}$ is given by the relation:

$$
t=\int \frac{d s}{v}=\int d y \frac{\sqrt{1+\left(\frac{d x}{d y}\right)^{2}}}{A+B y}=\int \frac{d y}{(A+B y) \sqrt{1-\Gamma^{2}(A+B y)^{2}}}
$$

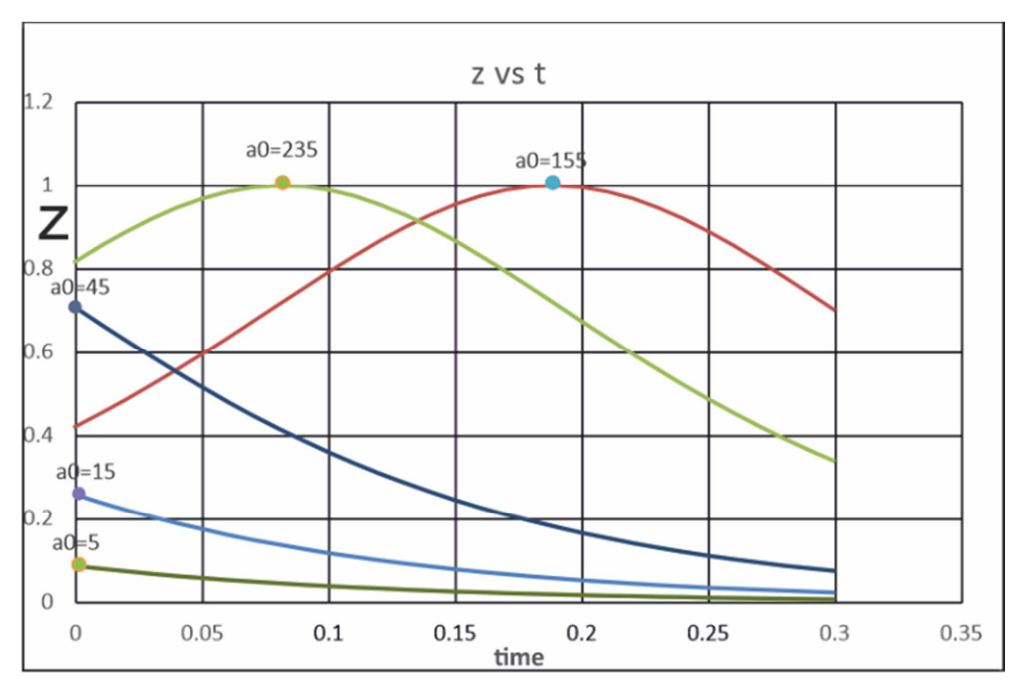

Figure 4. Time dependence of $Z$. Only the parts for $t>0$ concern the time.

$t=\int \frac{d z}{B z \sqrt{1-z^{2}}}=-\frac{1}{B} \ln \left(\frac{1+\sqrt{1-z^{2}}}{z}\right)$ so, by integrating between the limits $z_{0}$ and $\mathrm{z}$ we put $\frac{1+\sqrt{1-z_{0}^{2}}}{z_{0}}=\frac{1+2 \cos ^{2}\left(\frac{a_{9}}{2}\right)-1}{ \pm \sin z_{0}}=$ $\left|\cot \left(\frac{a_{0}}{2}\right)\right|=k$

$$
t=\int \frac{d z}{B z \sqrt{1-z^{2}}}=-\frac{1}{B} \ln \left(\frac{1+\sqrt{1-z^{2}}}{z}\right)+\frac{1}{B} \ln k=\frac{1}{B} \ln \left(\frac{k z}{1+\sqrt{1-z^{2}}}\right)
$$

By solving for $z$ we get

$$
\begin{gathered}
\exp (B t)=\frac{z k}{1+\sqrt{1-z^{2}}} \exp (B t)\left(\sqrt{1-z^{2}}\right)=z k-\exp (B t) \\
\mathrm{z}=\frac{2 k e^{B t}}{\left(k^{2}+\mathrm{e}^{2 \mathrm{Bt}}\right)}
\end{gathered}
$$

IF $z=1 t_{m}=\left|\frac{\ln (k)}{B}\right|$ which is the point where $\frac{d y}{d x}=0$. we get for $z=1$ the maximum value of $y$.

For $y<0 \mathrm{z}=\frac{2 k e^{-B t}}{\left(k^{2}+\mathrm{e}^{-2 \mathrm{Bt}}\right)}=\frac{2 e^{B t_{m}} e^{-B t}}{\left(e^{2 B t_{m}}+\mathrm{e}^{-2 \mathrm{Bt}}\right)}=\frac{2 e^{-B\left(t+t_{m}\right)}}{\left(1+\mathrm{e}^{-2 \mathrm{~B}\left(\mathrm{t}+\mathrm{t}_{\mathrm{m}}\right)}\right)}=\frac{2 e^{-B \xi}}{\left(1+\mathrm{e}^{-2 \mathrm{~B} \xi}\right)} \xi=t+t_{m}$

For $y>0 \mathrm{z}=\frac{2 k e^{B t}}{\left(k^{2}+\mathrm{e}^{2 \mathrm{Bt}}\right)}=\frac{2 e^{B t_{m}} e^{B t}}{\left(e^{2 B t_{m}}+\mathrm{e}^{2 \mathrm{Bt}}\right)}=\frac{2 e^{B\left(t-t_{m}\right)}}{\left(1+\mathrm{e}^{2 \mathrm{~B}\left(\mathrm{t}-\mathrm{t}_{\mathrm{m}}\right)}\right)}=\frac{2 e^{-B \xi}}{\left(1+\mathrm{e}^{-2 \mathrm{~B} \xi}\right)} \xi=t-t_{m}$

In the first case we have a simple calculation for $x$, but on the second we need to distinguish the $x$ for $t<=t_{m}$ and $t>t_{m}$.

For the time $t<=t_{m} x=-\frac{1}{B \Gamma} *\left(\operatorname{sign}\left(\sin \left(a_{0}\right)\right) * \sqrt{1-z^{2}}-\cos \left(a_{0}\right)\right)$ we get for $z=1$ 
$x_{m}=\frac{A}{B} \cot \left(a_{0}\right)$ and $y_{m}=\frac{A}{B}\left(\frac{1}{\left|\sin a_{0}\right|}-1\right)$ which are the coordinates of the highest point (point of reflection). This is valid for $a_{0}>\pi / 2$ or $a_{0}<3 \pi / 2$

For the time $t>t_{m} x=2 x_{m}+\frac{1}{(B \Gamma)}\left(\left(\operatorname{sign}\left(\sin \left(a_{0}\right)\right) * \sqrt{1-z^{2}}-\cos \left(a_{0}\right)\right.\right.$ this is due to the symmetry of $z$ so that $x\left(t_{m}+\right.$ $\xi)=x_{m}+\left(x_{m}-x\left(t_{m}-\xi\right)\right)=2 x_{m}-x\left(t_{m}-\xi\right)$

$$
z(-\xi)=\frac{2 e^{-B \xi}}{\left(1+\mathrm{e}^{-2 B \xi}\right)}=z(\xi)
$$

$$
\text { for } x=0 y=\frac{A l}{V l} *\left(e^{(V l * t)}-1\right) d y / d t=A l * e^{V l * t} \text { for } y>0 \text { or } y=\frac{A l}{V l} *\left(e^{(-V l * t)}-1\right)
$$

To calculate the wave fronts in excel we find for $-180<a_{0}<180$ the $\sin \left(a_{0}\right)$ and $\Gamma=\frac{\sin \left(a_{0}\right)}{A} E=1-\frac{\Gamma^{2}}{A}$

$z_{0}=\sin \left(a_{0}\right) k=a b s\left(\cot \frac{\left(a_{0}\right)}{2}\right) t_{m}=t_{\text {reflection }}=|\ln (k) / \beta|$ so for $k<1$ there is no reflection or if $a_{0}<2$ acot $(1)=\frac{\pi}{2}$ or $a_{0}>\frac{3 \pi}{2}$ then there is a reflection time and a maximum

$$
z=\frac{\left.2 * \operatorname{EXP}\left(\beta * \mid t-t_{-} \text {reflection }\right) \mid\right)}{1+\operatorname{EXP}\left(2 * \beta *\left|t-t_{\text {reflection }}\right|\right)} x_{\text {reflection }}=\frac{A}{\beta} \cot \left(a_{0}\right)
$$

To calculate $x$ we need to know if the $t_{\text {reflection }}$ is positive.

If it is negative or $t<t_{-}$reflection $\left.x=-1 /(\beta \Gamma)\left(\operatorname{SIGN}\left(\cos \alpha_{0}\right) * \sqrt{1-z^{2}}-\cos a_{0}\right)\right)$

If it is positive and then if $t>t_{\text {reflection }}$ then

$$
\mathrm{x}=2 * x_{-} \text {reflection }+\frac{1}{\beta * \Gamma} *\left(\operatorname{SIGN}\left(\cos \alpha_{0}\right) * \sqrt{\left.1-z^{2}\right)}-\cos a_{0}\right)
$$

\section{Ray from a Celestial Body to the Earth}

This problem was dealt by Newton who used a particle model of the light [14]

To find the path of light thar travels from moon (or any celestial body) and meets the atmosphere at a radius $r=$ $r_{g}+h_{\text {hatm }}$ where $r_{\mathrm{g}}$ is the radius of the glove and $\mathrm{h}_{\mathrm{atm}}$ is the height of the atmosphere the ideas presented in part 2 are applied. The problem can be solved for two cases: a) the speed is a linear function of $r-r_{g}$ and $b$ ) the speed is a function $(C+b r)^{\frac{1}{2}}[15]$ presents a treatment which is based in Lord's Rayleigh treatment.

\subsection{Speed Velocity as a Linear Function} ray comes at angle a with the ray (incidence angle) and the ray has an angle $\theta_{0}$ and the radius at this point $I r_{0}$ then by applying the Snell's law we get for another point located at (r, $\theta$ ) we have $\frac{\sin a_{0}}{c_{0}}=\frac{\sin a}{c}$ then if we assume that $c=c+b r$ so since $d s^{2}=c^{2} d t^{2}=d r^{2}+r^{2} d \theta^{2}$ so $\frac{\sin \alpha}{v}=K$
We consider a point located at the axis of a sphere. If the

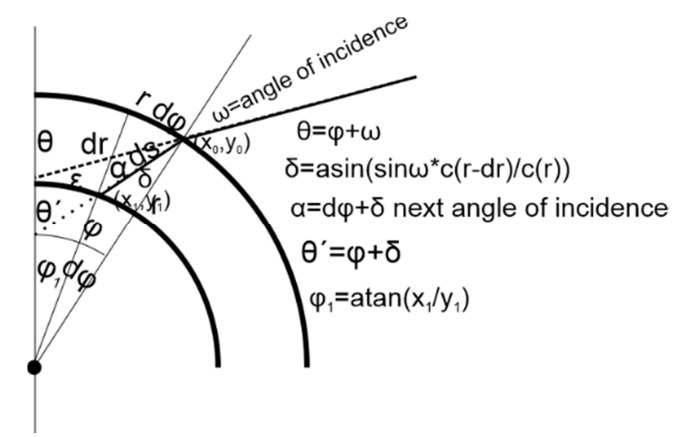

Figure 5. Step by step calculation of the ray.

On the upper limit of the atmosphere then $c=c_{0 u t}=1$ on the lower limit at $r=r_{g}=$ radius of earth, the speed will be $c_{0}=\frac{c_{0 u t}}{n_{\text {atm }}}$ We choose for the sake of the simulation to be $n_{\text {atm }}=1.05$ which is not the real value. We suppose that the speed decreases linearly as the light moves down the atmosphere from $c_{0 u t}=1$ at the outer limit of the atmosphere at the height $h=h_{\text {atm }}$ to the surface of the earth where $c=c_{\text {out }} / n_{\text {atm }} b=\frac{c_{\text {out }}\left(1-\frac{1}{n_{\text {atm }}}\right)}{h_{\text {atm }}}$

$$
\begin{aligned}
& c=\frac{c_{0 u t}}{n_{\text {atm }}}+\left(r-r_{g}\right) \frac{{ }^{c}{ }^{0 u t}\left(1-\frac{1}{n_{\text {atm }}}\right)}{h_{\text {atm }}} \text { for } r_{g}<=r<=r_{g}+h_{\text {atm }} c=c_{0 u t}=1 \text { for } r>r_{g}+h_{\text {atm }} \text { and } \\
& c=0: r<r_{g} c=\frac{c_{0 u t}}{n_{\text {atm }}}-r_{g} \frac{{ }^{c} \text { out }\left(1-\frac{1}{n_{\text {atm }}}\right)}{h_{\text {atm }}}+r \frac{{ }^{c}{ }_{\text {out }\left(1-\frac{1}{n_{\text {atm }}}\right)}}{h_{\text {atm }}} \text { so } C=\frac{c_{0 u t}}{n_{\text {atm }}}-r_{g} \frac{c_{0 u t}\left(1-\frac{1}{n_{\text {atm }}}\right)}{h_{\text {atm }}} \\
& r d \theta=d s \sin \alpha=d s K(c+b r) d \theta=\frac{(c+b r) d \tau K(c+b r)}{r}
\end{aligned}
$$

For a small time $\mathrm{d} \tau$ then we calculate the change of $\mathrm{r}$ : 
$d r=-(c+b r) \cos \alpha d \tau,-d \tau=\frac{d r}{(c+b r) \cos a}=\frac{d(c+b r)}{b(c+b r) \sqrt{1-K^{2}(C+b r)^{2}}}=\frac{d z}{b z \sqrt{1-K^{2} z^{2}}}=\frac{d K z}{b K z \sqrt{1-K^{2} z^{2}}}=\frac{\cos \omega d \omega}{b \sin \omega \cos \omega}$ so the integral with $z=1$ for $\tau=0$ is $-b \tau=\ln \left(\frac{1}{\sin \omega}-\frac{\cos \omega}{\sin \omega}\right)=\ln \left(\frac{1-\sqrt{1-K^{2} z^{2}}}{K z}\right)-\ln \left(\frac{1-\sqrt{1-K^{2}}}{K}\right)=\ln \left(\frac{1-\sqrt{1-K^{2} z^{2}}}{z\left(1-\sqrt{1-K^{2}}\right)}\right)$

$$
S O \mathrm{e}^{-b \tau}=\frac{1-\sqrt{1-K^{2} z^{2}}}{z\left(1-\sqrt{1-K^{2}}\right)}
$$

$$
Z=\frac{2\left(1-\sqrt{1-K^{2}}\right) e^{-b \tau}}{\left(\left(2-K^{2}-2 \sqrt{1-K^{2}}\right) e^{-2 b \tau}+K^{2}\right)}=\frac{2(1-\cos a) e^{-\beta t}}{(1-\cos a)^{2} e^{-2 b \tau}+\sin ^{2} a}
$$

For $\tau=0 \quad z=2 \frac{1-\sqrt{1-K^{2}}}{\left(\left(2-2 \sqrt{1-K^{2}}\right)\right)}=1$ then for $r=\frac{\frac{2\left(1-\sqrt{1-K^{2}}\right) e^{-b \tau}}{\left(\left(2-K^{2}-2 \sqrt{1-K^{2}}\right) e-2 b \tau+K^{2}\right)}-C}{b}=\frac{\frac{2(1-\cos a) e^{-b \tau}}{\left((1-\cos a)^{2} e^{-2 b \tau}+K^{2}\right)}-C}{b}$ So this relation gives as the radius $r$ as a function of time $\tau$. In excel we proceed from a radius $r_{1}$ to a radius $r_{2}$ in time $d \tau$ and then using geometry we find where the ray that starts at the circle with radius $r_{1}$ meets the circle at radius $r_{2}$. This method permits us to construct the ray.

$$
\begin{aligned}
& \text { since } c_{\text {out }}=1 \text { we get: } r(0)=\frac{1-C}{b}=\frac{1-\frac{c_{0 u t}}{n_{\text {atm }}}+r_{g} \frac{{ }^{c} \frac{{ }_{0 u t}\left(1-\frac{1}{n_{\text {atm }}}\right)}{h_{\text {atm }}}}{\frac{{ }_{\text {out }}\left(1-\frac{1}{n_{\text {atm }}}\right)}{h_{\text {atm }}}}}{r_{g}}+h_{\text {atm }} \\
& d \theta=\frac{(c+b r) d \tau K(c+b r)}{r K^{2}}=\frac{z^{2} d \tau}{r K}
\end{aligned}
$$

\subsection{Speed Velocity Depends on the Square Root of the Distance}

We proceed in a similar way as in case A:

$$
\begin{gathered}
d r=-\sqrt{(c+b r)} \cos \alpha d \tau,-d \tau=\frac{d r}{\sqrt{(c+b r)} \cos a}=\frac{d(c+b r)}{b \sqrt{(c+b r)} \sqrt{1-K(c+b r)}}=\frac{d z}{b z \sqrt{1-K z}} \tau=\int_{1}^{Z} \frac{d z}{b z \sqrt{1-K x}} \\
\left.=\frac{2}{\sqrt{K}}\left(\arctan \left(\frac{K z}{1-K z}\right)-\arctan \left(\frac{K}{1-K}\right)\right)=\frac{2}{\sqrt{K}} \arctan \left(\frac{\frac{K z}{1-K z}-\frac{K}{1-K}}{1+\frac{K^{2} z}{(1-K z))(1-K)}}\right)=\frac{2}{\sqrt{K}} \arctan \left(\frac{K(z-1)}{1-K z+2 K^{2} z}\right)\right) \\
\tan \left(\frac{\sqrt{K} \tau}{2}\right)=\frac{K(z-1)}{z K(K-1)} z K(K-1) \tan \psi=K z-K \\
z((K-1) \tan \psi-1)=-1 z=-\frac{1}{(K-1) \tan \psi-1} \text { where } \psi=\frac{\sqrt{K} \tau}{2} \\
r=\frac{-\frac{1}{(K-1) \tan \psi-1}-C_{s}}{b_{S}}
\end{gathered}
$$

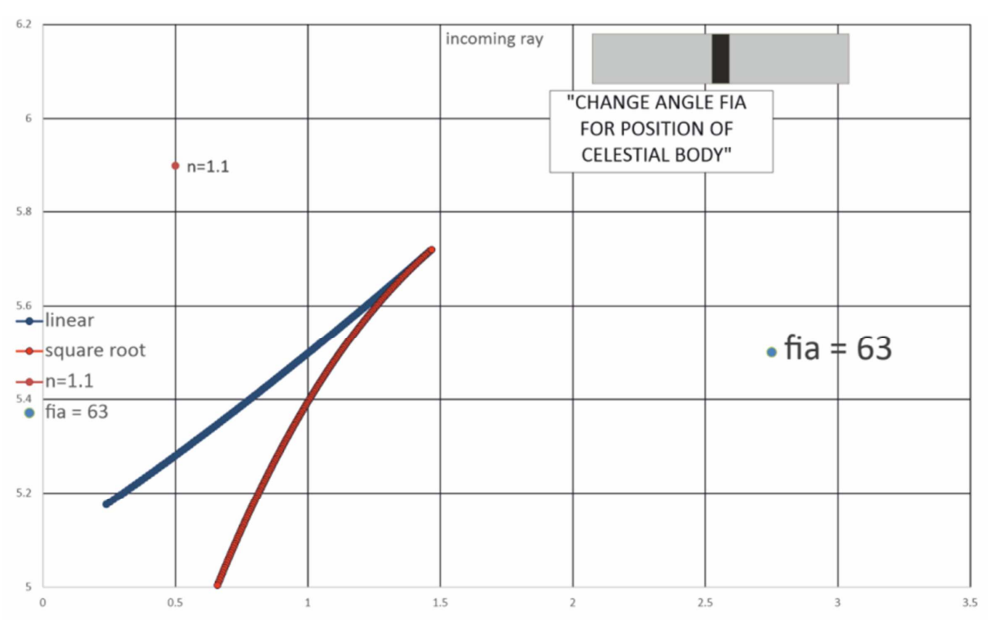

Figure 6. The deviation of the ray for 2 models. 
As can be seen on figure 6 the tangent of the curve at the lowest point determines the "height" where the celestial body is seen. This means that different models give different "apparent" position of the celestial body [13].

\section{Conclusion}

The use of Excel files can help the students to connect the rays with the movement of the wave fronts. The simple mathematical treatment of this subject makes it very well suited for using in introductory undergraduate physics. The user can use "scroll bars" to change the time so that the wave fronts can be seen as moving along the screen. The students can appreciate that the bigger distance between wave front corresponds to bigger wave speeds and vice versa. By treating the incoming rays from a celestial body, the students can understand that the tangent of the rays on the position of the observer, determines the position that this body is observed. Also, can understand that even if the celestial body (as the sun) is under the Horizon, the refraction can cause the body to be seen.

\section{Appendix: Geometry of the Earth - Moon}

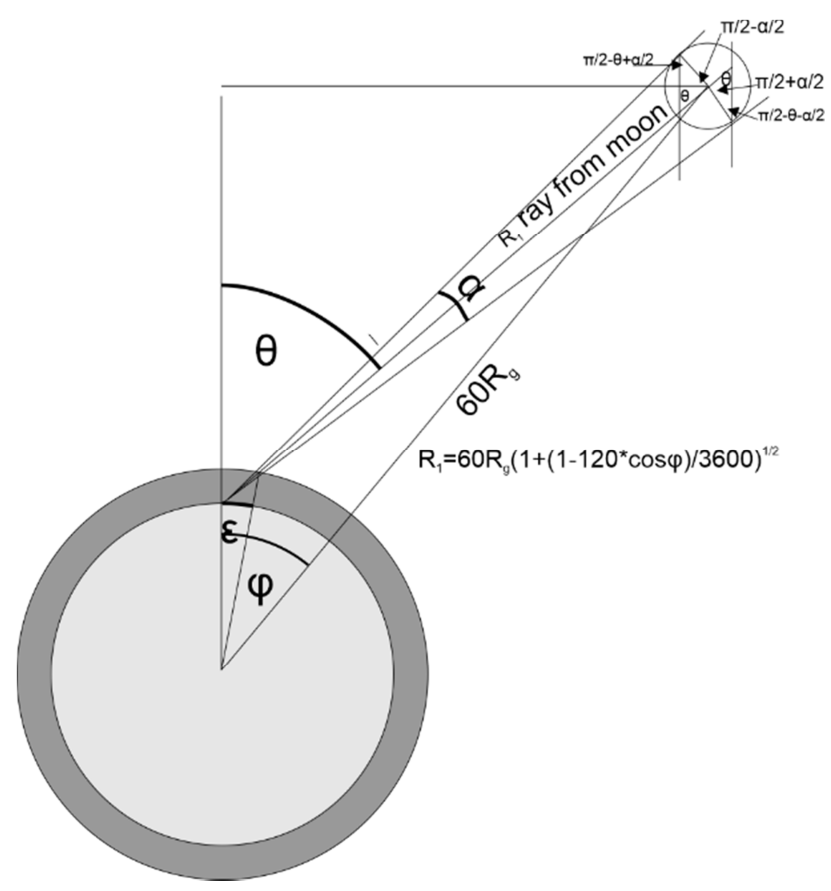

Figure 7. Geometry of the problem.

Distance between the pole and the center of the $K_{1} K_{2}$ is seen by an angle $\alpha=0,5^{\circ}$, so can calculate the coordinates of the limits of the visible moon on the basis of the angle

To find the tangent of the moon from the pole we put:

$\left(y-y_{S}\right)^{2}+\left(x-x_{S}\right)^{2}=R_{S}^{2} y=R g+x t$ where $\mathrm{t}$ is the tangent. The equation for $\mathrm{x}$ is: $X^{2}\left(1+t^{2}\right)+2 x\left(D_{y} t-\right.$ $\left.x_{S}\right)+x_{S}^{2}+D_{y}^{2}-R_{S}^{2}=0 D_{y}=R_{g}-y_{s}$ for finding the tangent the equation should have a double root which means that its determinant should be zero or:

$t^{2}\left(R_{s}^{2}-x_{s}^{2}\right)-2 D_{y} x_{S} t+R_{S}^{2}-D_{y}^{2}=0$ so we find two roots

$$
\begin{aligned}
& t_{1,2}=\frac{D_{y} x_{S} \pm R_{S} \sqrt{D_{y}^{2}+x_{S}^{2}-R_{S}^{2}}}{R_{S}^{2}-x_{S}^{2}} \text { So, we find for the } 2 \text { points: } \\
& x=-\frac{D_{y} t-x_{S}}{1+t^{2}} \\
& \theta=\operatorname{atan}\left(\frac{R_{G S} \sin \varphi}{R_{g s} \cos \varphi-R_{g}}\right) \text { If } R_{g} \text { the earth's radius, } h_{\text {atm }} \text { is the }
\end{aligned}
$$
height of the atmosphere

$R_{g 2}=R_{g}+h_{\text {atm }}$ the total radius. The same is applied also between 2 radii

$$
R_{1}=r, R_{2}=r-d r
$$

If the ray who starts from the moon makes an angle $\theta$ with the $y$-axis then if the ray meets the limit of the atmosphere at a point making an angle $\varepsilon$ with the $y$-axis. The incidence angle is $\theta-\varepsilon$ To find the point of incidence we solve the system of equations: $y=R_{g}+\cot \theta x$ and $x^{2}+y^{2}=R_{g 2}^{2}$ we get $x=\left(-R_{g} \cos \theta+\sqrt{R_{g 2}^{2}-R_{g}^{2} \sin \theta^{2}}\right) \sin \theta$

which gives us the angles $\theta$ through which the rays from the moon are seen from the pole.

with the $y$-axis, then the incidence angle is $\omega-\varphi_{1}$ and the angle of refraction is found from $\sin ($ diathl $)=$ $\frac{\sin (\text { incidenc) }}{n(r-d r)} n(r)=\frac{\sin (\text { incidecne })(c+b(r-d r))}{c+b r}$ we find the angle $\varepsilon$ from tanc $=x / y$ and then we proceed to find next point of intersection of the refracted ray with circle of radius $r$-dr. Now the new refracted ra is making an angle

$\theta=\varepsilon+$ diath where diath is the angle of refraction

Now the set of equations $y=y_{0}+\cot \theta\left(x-x_{0}\right), y^{2}+$ $x^{2}=(r-d r)^{2}$ We get for the

$$
\begin{gathered}
\text { Equation } y_{0}^{2}+\cot ^{2} \theta\left(x^{2}-2 x x_{0}+x_{0}^{2}\right)+2 y_{0}(x- \\
\left.x_{0}\right) \cot \theta+x^{2}-R_{1}^{2}=0 \text { or } \\
\begin{array}{c}
x^{2}\left(1+\cot ^{2} \theta\right)+2 x \cot \theta\left(y_{0}-x_{0} \cot \theta\right)+\left(y_{0}-x_{0} \cot \theta\right)^{2} \\
-R_{1}^{2}=0 \\
\mathrm{Da}=\cot ^{2} \theta\left(y_{0}-x_{0} \cot \theta\right)^{2}-\left(y_{0}-x_{0} \cot \theta\right)^{2}(1+ \\
\left.\cot ^{2} \theta\right)+R_{1}^{2}\left(1+\cot ^{2} \theta\right)=\frac{R_{1}^{2}}{\sin ^{2} \theta}-\left(y_{0}-x_{0} \cot \theta\right)^{2}= \\
\left(R_{1}^{2}-\left(y_{0}-x_{0} \cot \theta\right)^{2} \sin ^{2} \theta\right) / \sin ^{2} \theta \\
x=\left(-\left(y_{0}-x_{0} \cot \theta\right) \cos \theta+\sqrt{D}\right) \sin \theta \text { where } D= \\
(r-d r)^{2}-\left(y_{0}-x_{0} \cot \theta\right)^{2} \sin { }^{2} \theta
\end{array}
\end{gathered}
$$

\section{References}

[1] Halloun, I. (2018). Scientific Models and Modeling in the Framework of Systemic Cognition and Education. Working paper. Jounieh, LB: H Institute.

[2] Sunil Kumar Katoch, (2020) "MS-Excel Spreadsheet Applications in Introductory Under-Graduate Physics-A Review", Journal of Science and Technology, Vol. 05, Issue 03 , May-June 2020, pp48-52. 
[3] Pavlos Mihas (2017) Excel Files for Teaching Two Dimensional Motions and their Curvature, Global Journal of Science Frontier Research: I Interdisciplinary vol. 16.3GJSFR (2016) Volume 16 Issue 3: 23-32 January 2017 DOI: 10.17406/GJSFRIVOL16IS3PG23.

[4] Pavlos Mihas (2020) Original Paper, Excel Files for Newton's Proposition V, May 2020, Applied Science and Innovative Research 4 (2): 41-52 DOI: 10.22158/asir.v4n2p41.

[5] Manuel I González (2018) Lens ray diagrams, Phys. Educ. 53 03501 .

[6] Pavlos Mihas (2019) Software for Teaching through Interactive Demonstrations about Converging Lenses, Applied Science and Innovative Research (Online) Vol. 3, No. 1, 2019 www.scholink.org/ojs/index.php/asir.

[7] Pavlos Mihas (2021) Excel Files for Teaching Caustics of Rainbow and Lenses (spherical, Huygens and Ibn Sahl) in Journal of Physics \& Optics Sciences. SRC/JPSOS/147 March 2021 DOI: 10.47363/JPSOS/2021(3)13.

[8] Mila Kryjevskaia, MacKenzie R. Stetzer, and Paula HR. L. Heron (2012) Student understanding of wave behavior at a boundary: The relationships among wavelength, propagation speed, and frequency, American Journal of Physics80 (4), April 2012.

[9] Tutorials in Introductory Physics by Peter S. Shaffer McDermott, Lillian C | Jan 1, 2012.

[10] Pavlos Mihas \& Triantafyllos Gemousakakis (2020) Teaching about waves with applications in lenses, Journal of Education and Social studies, Vol. 2,2 pp 38-52.

[11] G. Polya (1954) Induction and Analogy in Mathematics, Vol. 1 Princeton University Press.

[12] Leonid Minkin \& Percy Whitning (2019) Restricted Brachistochrone, The Physics Teacher 57, 359.

[13] Brian S. Blais (2020) Model Comparison in the introductory Laboratory, The Physics Teacher 58, 209.

[14] Michael Nauenberg (2017) Newton's theory of the atmospheric refraction of light American Journal of Physics 85, 921 (2017).

[15] W. J. Humphreys (1920) Physics of the Air THE FRANKLIN INSTITUTE OF THE STATE OF PENNSYLVANIA By J. B. LIPPINCOTT COMPANY, 2012 printing by FORGOTTEN BOOKS www.forgottenbooks.org. 